\title{
Altered intrinsic regional brain activity in male patients with severe obstructive sleep apnea: a resting-state functional magnetic resonance imaging study
}

\author{
De-Chang Peng' \\ Xi-Jian Dai ${ }^{1,2}$ \\ Hong-Han Gong' \\ Hai-Jun Li' \\ Xiao $\mathrm{Nie}^{\prime}$ \\ Wei Zhang ${ }^{3}$
}

'Department of Radiology, The First Affiliated Hospital of Nanchang University, Jiangxi, ${ }^{2}$ Department of Imaging and Interventional Radiology, Prince of Wales Hospital, The Chinese University of Hong Kong, Shatin,

Hong Kong, Special Administrative Region, ${ }^{3}$ Department of Pneumology, The First Affiliated Hospital

of Nanchang University, Jiangxi, People's Republic of China

Video abstract

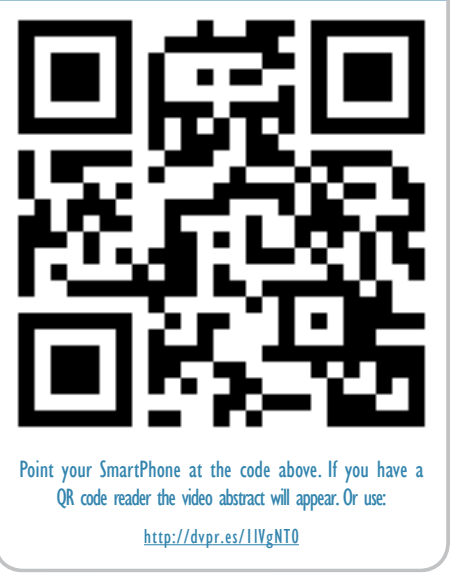

Correspondence: Wei Zhang

Department of Pneumology, The First Affiliated Hospital of Nanchang University, 17 Yong Wai

Zheng Street, DongHu District, Nanchang City, Jiangxi 330006, People's Republic of China

Tel +86 79l 88692582

Email zhangweiliuxin@I63.com
This article was published in the following Dove Press journal:

Neuropsychiatric Disease and Treatment

19 September 2014

Number of times this article has been viewed

Background: Previous studies have demonstrated that obstructive sleep apnea (OSA) is associated with abnormal brain structural deficits. However, little is known about the changes in local synchronization of spontaneous activity in patients with OSA. The primary aim of the present study was to investigate spontaneous brain activity in patients with OSA compared with good sleepers (GSs) using regional homogeneity (ReHo) analysis based on resting-state functional magnetic resonance imaging (MRI).

Methods: Twenty-five untreated male patients with severe OSA and 25 male GSs matched for age and years of education were included in this study. The ReHo method was calculated to assess the strength of local signal synchrony and was compared between the two groups. The observed mean ReHo values were entered into Statistical Package for the Social Sciences software to assess their correlation with behavioral performance.

Results: Compared with GSs, patients with OSA showed significantly lower ReHo in the right medial frontal gyrus (BA11), right superior frontal gyrus (BA10), right cluster of the precuneus and angular gyrus (BA39), and left superior parietal lobule (BA7), and higher ReHo in the right posterior lobe of the cerebellum, right cingulate gyrus (BA23), and bilateral cluster covering the lentiform nucleus, putamen, and insula (BA13). The lower mean ReHo value in the right cluster of the precuneus and angular gyrus had a significant negative correlation with sleep time ( $r=-0.430, P=0.032$ ), and higher ReHo in the right posterior lobe of the cerebellum showed a significant positive correlation with stage 3 sleep $(r=0.458, P=0.021)$ and in the right cingulate gyrus showed a significant positive correlation with percent rapid eye movement sleep $(r=0.405, P=0.045)$.

Conclusion: Patients with OSA showed significant regional spontaneous activity deficits in default mode network areas. The ReHo method is a useful noninvasive imaging tool for detection of early changes in cerebral ReHo in patients with OSA.

Keywords: obstructive sleep apnea, regional homogeneity, intermittent hypoxia, functional magnetic resonance imaging, resting state, spontaneous activity

\section{Introduction}

Obstructive sleep apnea (OSA) is a common sleep disorder and is characterized by repeated episodes of partial (hypopnea) or complete (apnea) obstruction of the upper airway during sleep due to pharyngeal collapse, resulting in recurrent chronic intermittent hypoxia and fragmentation of sleep. ${ }^{1}$ Population-based epidemiological studies indicate that OSA affects at least 3\%-7\% of middle-aged adult men, $2 \%-5 \%$ of women, and up to $4 \%$ of children. ${ }^{2-6}$ OSA may cause excessive daytime sleepiness, frequently altered 
cardiopulmonary function, independently increased risk for hypertension, insulin resistance, cardiovascular disease, and motor vehicle accidents. ${ }^{7-10}$ For patients, OSA can result in deficits in executive function, memory, coordination of movement, ${ }^{11}$ and attention. ${ }^{12}$ Although the main contributory factors are presumed to be sleep fragmentation and intermittent nocturnal hypoxemia during sleep apneas, ${ }^{13}$ the pathological mechanism of the brain deficits is still unclear.

To date, limited work has been done to investigate OSA within a neuroscientific framework, and only a handful of studies have directly addressed the neural basis for the OSA trait in patients. Previous neuroimaging studies have documented altered gray matter volume deficits in the hippocampus, ${ }^{14-16}$ temporal lobe, frontal lobe, anterior cingulate cortex, and cerebellar regions ${ }^{17,18}$ in patients with OSA using the voxel-based morphometry method. Moreover, diffusion tensor imaging studies show a wide range of white matter integrity changes within the corpus callosum, frontal cortex, temporal cortex, parietal cortices, cingulum bundle, and cerebellum. ${ }^{19-21}$ Magnetic resonance spectroscopy is also a useful neuroimaging tool because it provides a measure of changes in cerebral metabolism that may reflect pathological insults to brain integrity. Several studies using magnetic resonance spectroscopy have demonstrated significant metabolic changes in OSA. ${ }^{22-24}$ Although these studies have focused on structural and metabolic changes in the brains of patients with OSA, little research has been undertaken to examine how the human brain is affected by OSA, leaving the mechanisms unclear.

Functional magnetic resonance imaging (MRI), a noninvasive, high spatial, and temporal resolution technique, can reflect the intrinsic functional organization of the human brain. ${ }^{25}$ Previous functional MRI studies of OSA have mainly focused on task studies and more employ either respiratory or cognitive challenges, significant changes are found in brain activation levels compared with good sleepers (GSs). ${ }^{26-28}$ In recent years, resting-state functional MRI has become a hot topic in neuroimaging studies. Regional homogeneity (ReHo), a newly developed resting-state functional MRI approach, can analyze the similarities and coherence of intraregional spontaneous low-frequency $(<0.08 \mathrm{~Hz})$ signal fluctuations using voxel-wise analysis across the whole brain. ReHo is based on a data-driven approach, so does not require the onset time of the stimulus or prior knowledge, and has good test-retest reliability. The simple calculations involved and reliable characterization make it a potentially useful tool for analysis of resting-state functional MRI data, and ReHo could be regarded as a measure for investigating whether local synchronization of spontaneous brain activity is associated with OSA trait. ReHo has been used successfully to detect local abnormalities in subjects with various neurological diseases, including sleep deprivation, ${ }^{29}$ transient ischemic attacks, ${ }^{30}$ schizophrenia, ${ }^{31}$ Parkinson's disease,${ }^{32}$ and epilepsy. ${ }^{33}$ Using ReHo, a significant pattern of abnormal local cortical and subcortical connectivity has been shown in patients with OSA. ${ }^{34}$ However, resting-state functional MRI data are still scarce in patients with the condition, so more research to reveal the neuropathophysiology consequences of OSA would be of great significance.

In this prospective study, we hypothesized that, compared with GSs, patients with OSA would exhibit abnormal ReHo activity in at least parts of the areas, based on the previous studies reviewed, including the prefrontal lobe, temporal lobe, hippocampus, and cerebellum. To test this hypothesis, we used the ReHo method to investigate whether the synchrony of regional spontaneous brain activity on resting-state functional MRI was altered in male patients with severe OSA when compared with GSs. This comparison is important in OSA studies because sleepiness and oxygen deficit increases in the supine position and is expected to be accentuated following OSA.

\section{Materials and methods Subjects}

Twenty-five untreated male patients with severe OSA and 25 male GSs matched for age and years of education were recruited for this study from the Sleep Monitoring Room in the Respiratory Department of The First Affiliated Hospital of Nanchang University. Each candidate had a detailed clinical interview and physical examination, completed a sleep questionnaire, and underwent overnight polysomnography. Inclusion criteria for the patients with OSA were as follows: male gender; age older than 22 years but younger than 60 years; and an apnea-hypopnea index (AHI) $>30$. Exclusion criteria for both patients and GSs were as follows: other sleep disorders, such as insomnia and a sleep-related eating disorder; history of cardiovascular disease, hypothyroidism, or diabetes mellitus; left-handedness; alcohol or illicit drug abuse, or current intake of psychoactive medications; a central nervous system disorder (neurodegenerative disease, epilepsy, head injury, psychosis, current depression); and contraindications to MRI, such as claustrophobia, metallic implants, or devices in the body. All subjects underwent a cognitive assessment using the Montreal Cognitive Assessment administered by two independent neuropsychologists. This study was approved by the human research ethics committee at The First Affiliated Hospital of Nanchang University, and all participants provided their written informed consent. 


\section{Polysomnography}

We used standard overnight polysomnography with the LESeries physiological monitoring system (Alice $5 \mathrm{LE}$, Respironics, Orlando, FL, USA) in the sleep center of our hospital, along with standard electroencephalogram, electrooculogram, chin electromyogram, and electrocardiogram checks simultaneously. Ventilatory flow at the nose and mouth was measured with thermistors. Ventilatory movements of the chest and abdomen were monitored by inductive plethysmography bands. Arterial oxygen saturation was measured transcutaneously by fingertip pulse oximetry. Apnea was defined as continuous cessation of airflow for more than 10 seconds and hypopnea was defined as a decrease in airflow by more than $30 \%$ with arousal or oxygen desaturation more than $4 \% .35,36$ The AHI was determined by dividing the average of the total number of apnea/hypopnea events by the estimated hours of sleep. Subjects were recorded on videotape and were continuously observed by a polysomnography technician. Polysomnography was performed from $10 \mathrm{pm}$ to 6 am next morning.

\section{MRI parameters}

MRI scanning was performed on a 3-Tesla MR scanner (Siemens, Munich, Germany). The scans consisted of a location image, T1-weighted sagittal images, T2WI axial/coronal images, and functional images. For the T1 sagittal images, 176 images were obtained (repetition time $1,900 \mathrm{msec}$, echo time $2.26 \mathrm{msec}$, thickness $1.0 \mathrm{~mm}$, gap $0.5 \mathrm{~mm}$, acquisition matrix $256 \times 256$, field of view $250 \mathrm{~mm} \times 250 \mathrm{~mm}$, flip angle 9 degrees). Finally, at the same position, 240 functional images were obtained (repetition time $2,000 \mathrm{msec}$, echo time $30 \mathrm{msec}$, thickness $4.0 \mathrm{~mm}$, gap $1.2 \mathrm{~mm}$, acquisition matrix $64 \times 64$, flip angle 90 degrees, field of view $230 \mathrm{~mm} \times 230 \mathrm{~mm}$, 30 axial slices with gradient-recalled echo-planar imaging pulse sequence covering the whole brain).

\section{Functional MRI data analysis}

Functional data were checked using MRIcro software (www. MRIcro.com) to exclude defective data. The first ten time points for the functional images were discarded due to the possible instability of the initial MRI signal and the participants' adaptation to the scanning environment. On the basis of MATLAB2010a software (Mathworks, Natick, MA, USA), the rest of the data preprocessing was performed using DPARSFA software (http://rfmri.org/DPARSF), including DICOM form transformation, slice timing, head motion correction, and spatial normalization. Motion time courses were obtained by estimating the values for translation $(\mathrm{mm})$ and rotation (degrees) for each subject. Participants who had more than $1.5 \mathrm{~mm}$ maximum displacement in $\mathrm{x}, \mathrm{y}$, or $\mathrm{z}$ and
1.5 degrees of angular motion during whole functional MRI scans were rejected. The six Friston head motion parameters was used to correct for the effects of head motion based on recent work showing that higher-order models are more effective in removing head motion effects. ${ }^{37,38}$ After correction for head motion, the functional MRI images were spatially normalized to the Montreal Neurological Institute space using the standard echo-planar imaging template in Statistical Parametric Mapping 8 (http://www.fil.ion. ucl.ac.uk/spm) software and resampling the images at a resolution of $3 \mathrm{~mm} \times 3 \mathrm{~mm} \times 3 \mathrm{~mm}$. After preprocessing, the time series for each voxel were detrended and filtered (bandpass $0.01-0.08 \mathrm{~Hz}$ ) to reduce low-frequency drift and high frequency physiological respiratory and cardiac noise and time series linear detrending. ReHo computation based on protocols from previous studies was performed with REST software (http://www.resting-fmri. Sourceforge.net). ${ }^{29}$

Individual ReHo maps were generated by calculating Kendall's coefficient of concordance (KCC) for the time series of a given voxel with those of its nearest neighbors (26 voxels) in a voxel-wise analysis. The computational formula was ${ }^{39}$

$$
\mathrm{ReHo}=\frac{\sum\left(R_{i}\right)^{2}-n(\bar{R})^{2}}{K^{2}\left(n^{3}-n\right) / 12},
$$

where ReHo is the $\mathrm{KCC}$ among the given voxels, ranging from 0 to 1 ; when a given cluster and its adjacent cluster in a time series is more consistent, the $\mathrm{KCC}$ value is more close to 1 . $K$ is the voxel number of time series within a measured cluster (the smallest unit of measured ReHo, comprised of more adjacent clusters); here, $K=27$ (one given voxel which was located in the cubic center plus its adjacent 26 voxels); $n$ is the number of ranks; $R_{i}$ is the sum rank of the ith time point, where $\bar{R}=(n+1) K / 2$ is the mean of the $R$ values; the standard ReHo value is the ReHo value of each cluster/mean of the whole brain ReHo value; thus, the individual ReHo map was generated for each dataset. To reduce the influence of individual variations in $\mathrm{KCC}$ value normalization of the ReHo maps was done by dividing the KCC for each voxel by the average KCC of the whole brain. The resulting functional MRI data were then spatially smoothed with a Gaussian kernel of $6 \times 6 \times 6 \mathrm{~mm}^{3}$ full-width at half-maximum.

\section{Statistical analysis}

One-sample $t$-tests were first performed within each group to detect where the standardized $\mathrm{KCC}$ values were larger than the 
global mean KCC. The between-conditions statistical threshold was set at a cluster size of $P<0.001$, voxels at $P<0.001$, and a cluster volume $\geq 1,080 \mathrm{~mm}^{3}$ (a minimum continuous cluster volume of $1,080 \mathrm{~mm}^{3}$ ), corrected by the false discovery rate. Group differences based on the ReHo method were investigated using a two-samples $t$-test in the REST tool kit. The ages were included as nuisance covariates (variables of no interest) to remove the residual effect in the statistical models applied. A corrected significance level of individual voxel $P<0.001$ and cluster volume $\geq 1,080 \mathrm{~mm}^{3}$, using a false discovery rate-corrected threshold of $P<0.05$, was used to determine the between-conditions statistical significance. The results were presented by Xjview and REST.

\section{Brain-behavior correlation analysis}

Based on our ReHo findings, we identified brain regions that demonstrated significant between-group differences. These regions were classified as regions of interest and saved as masks with REST software. For each region of interest, the mean ReHo value was extracted by averaging ReHo values over all voxels for each patient with OSA. Finally, the mean ReHo values were entered into Statistical Package for the Social Sciences version 17.0 software (SPSS Inc, Chicago, IL, USA) to calculate their correlation with behavioral performance.

\section{Results}

\section{Demographic and clinical data}

The demographic and clinical characteristics of each group are summarized in Table 1 . The patients with OSA had significant higher scores for body mass index $(t=6.25$, $P<0.001)$, AHI $(t=15.51, P<0.001), \mathrm{SpO}_{2}<90 \%(t=6.66$,
$P<0.001)$, and Epworth Sleepiness Scale $(t=7.64, P<0.001)$ when compared with GSs.

\section{Changes in ReHo in OSA compared with GSs}

Compared with GSs, patients with OSA showed a significantly lower ReHo in the right medial frontal gyrus (BA11), the right superior frontal gyrus (BA10), the right cluster of the precuneus and angular gyrus (BA39), and the left superior parietal lobule (BA7), and a significantly higher ReHo in the right posterior lobe of the cerebellum, the right cingulate gyrus (BA23), and the bilateral cluster covering the lentiform nucleus, putamen, and insula (BA13). The details are presented in Figure 1 and Table 2. The mean ReHo value of these altered areas were extracted (Figure 2).

\section{Correlation analysis}

In patients with OSA, the AHI score showed a significant positive correlation with arousal index $(r=0.642, P=0.001)$ and a negative correlation with percent time in rapid eye movement sleep ( $r=-0.429, P=0.032)$ and Montreal Cognitive Assessment score $(r=-0.405, P=0.045)$. Percent time in stage 2 sleep showed a negative correlation with percent time in rapid eye movement sleep $(r=-0.584, P=0.002)$ and Epworth Sleepiness Scale score $(r=-0.531, P=0.006)$. Body mass index showed a significant positive correlation with arousal index ( $r=0.582, P=0.002)$.

The observed higher mean ReHo signal values in the right posterior lobe of the cerebellum showed a significant positive correlation with percent time in stage 3 sleep $(r=0.458$, $P=0.021$ ), the right cingulate gyrus showed a significant

Table I Demographic and clinical characteristics of patients with OSA and GSs

\begin{tabular}{|c|c|c|c|c|c|}
\hline \multirow[t]{2}{*}{ Characteristics } & \multicolumn{2}{|c|}{ Patients with OSA $(n=25)$} & \multicolumn{2}{|c|}{ GSs $(n=25)$} & \multirow[t]{2}{*}{$P *$} \\
\hline & Mean & SD & Mean & SD & \\
\hline Age, years & 39.4 & 1.7 & 39.5 & 1.6 & 0.946 \\
\hline Education, years & 11.9 & 3.1 & 10.8 & 3.8 & 0.258 \\
\hline BMI, $\mathrm{kg} / \mathrm{m}^{2}$ & 27.8 & 3.4 & 22.9 & 2.1 & $<0.001$ \\
\hline Total sleep time, minutes & 373.6 & 31.8 & 397.4 & 24.9 & 0.005 \\
\hline $\mathrm{AHI}$, per hour & 60.0 & 18.6 & 2.2 & 1.3 & $<0.001$ \\
\hline $\mathrm{NI} \%$ & 32.2 & 20.4 & 10.6 & 4.0 & $<0.001$ \\
\hline $\mathrm{N} 2 \%$ & 45.2 & 17.4 & 48.6 & 6.4 & 0.361 \\
\hline N3\% & 14.2 & 9.3 & 21.0 & 5.2 & $<0.001$ \\
\hline REM\% & 8.4 & 8.6 & 20.4 & 6.9 & $<0.001$ \\
\hline $\mathrm{SpO}_{2}<90 \%$ & 26.5 & 19.7 & 0.2 & 0.2 & $<0.001$ \\
\hline Arousal index per hour & 44.0 & 23.3 & 11.8 & 3.0 & $<0.001$ \\
\hline ESS & 15.2 & 7.3 & I.I & I.I & $<0.001$ \\
\hline $\mathrm{MoCA}$ & 25.7 & 2.3 & 27.1 & 2.3 & 0.036 \\
\hline
\end{tabular}

Notes: $* P$, independent-samples $t$-tests. $P<0.05$.

Abbreviations: AHI, apnea-hypopnea index; BMI, body mass index; ESS, Epworth Sleepiness Scale; OSA, obstructive sleep apnea; GSs, good sleepers; REM, rapid eye movement; SD, standard deviation; $\mathrm{SpO}_{2}<90 \%$, percentage of total sleep time spent at oxygen saturations less than $90 \%$; NI\%, N2\%, N3\%, percent of total sleep time at diagnostic polysomnography spent irrelevant stages; MoCA, Montreal Cognitive Assessment. 
Table 2 Two-samples t-test differences in ReHo method between OSA and GSs

\begin{tabular}{|c|c|c|c|c|c|c|c|c|c|}
\hline \multirow{2}{*}{$\begin{array}{l}\text { Conditions } \\
\text { OSA > GSs }\end{array}$} & \multirow{2}{*}{$\begin{array}{l}\text { Brain regions of peak } \\
\text { coordinates } \\
\text { Cerebellum posterior lobe } \\
\text { (declive, culmen) }\end{array}$} & \multirow{2}{*}{$\begin{array}{l}R / L \\
R\end{array}$} & \multirow{2}{*}{$\begin{array}{l}\text { BA } \\
-\end{array}$} & \multirow{2}{*}{$\begin{array}{l}\mathbf{V}\left(\mathrm{mm}^{3}\right) \\
432\end{array}$} & \multirow{2}{*}{$\begin{array}{l}\begin{array}{l}t \text {-score of } \\
\text { peak voxel }\end{array} \\
4.5437\end{array}$} & \multicolumn{3}{|c|}{$\begin{array}{l}\text { MNI coordinates } \\
\text { of peak voxel }\end{array}$} & \multirow{2}{*}{$\begin{array}{l}\boldsymbol{P} \text {-values } \\
<0.001\end{array}$} \\
\hline & & & & & & 3 & -69 & -18 & \\
\hline OSA $>$ GSs & Lentiform nucleus, putamen, insula & L & 13 & 2,160 & 4.9744 & -27 & -15 & 3 & $<0.001$ \\
\hline OSA $>$ GSs & Lentiform nucleus, putamen, insula & $\mathrm{R}$ & 13 & 4,536 & 5.4834 & 33 & -3 & 3 & $<0.001$ \\
\hline OSA $>$ GSs & Cingulate gyrus & $\mathrm{R}$ & 23 & 675 & 4.3834 & 0 & -6 & 42 & $<0.00$ I \\
\hline OSA $<$ GSs & Medial frontal gyrus & $\mathrm{R}$ & 11 & 837 & -4.927 & 9 & 54 & -15 & $<0.001$ \\
\hline OSA $<$ GSs & Superior frontal gyrus & $\mathrm{R}$ & 10 & 567 & -4.9346 & 18 & 72 & 3 & $<0.00$ I \\
\hline OSA $<$ GSs & Precuneus, angular gyrus & $\mathrm{R}$ & 39 & 540 & $-4.48 I I$ & 42 & -72 & 36 & $<0.001$ \\
\hline OSA $<$ GSs & Superior parietal lobule & L & 7 & 594 & -4.4816 & -33 & -69 & 54 & $<0.001$ \\
\hline
\end{tabular}

Notes: The between-condition statistical threshold was set at cluster size with $P<0.05$, voxel with $P<0.00 \mathrm{I}$, and $V \geq 405 \mathrm{~mm}^{3}$, corrected for false discovery rate (FDR). Abbreviations: R, right; L, left; BA, Brodmann's area; V, volume; MNI, Montreal Neurological Institute; GSs, good sleepers; OSA, patients with obstructive sleep apnea; ReHo, regional homogeneity.

positive correlation with percent time in rapid eye movement sleep ( $r=0.405, P=0.045$ ), and a lower mean ReHo in the right cluster of the precuneus and angular gyrus showed a significant negative correlation with sleep time $(r=-0.430, P=0.032)$.

\section{Discussion}

Our previous study using the ReHo method demonstrated that many brain areas show obvious sex differences, both during the rested wakeful condition and the sleep deprivation condition. ${ }^{29}$ To avoid the influence of sex differences, we chose only male patients with OSA to participate in this research. To our knowledge, few resting-state functional MRI studies have been conducted in patients with OSA. ${ }^{34}$ The present study used ReHo to investigate the synchrony of regional spontaneous activity in resting-state functional MRI among patients with OSA. Consistent with our hypothesis, patients with OSA showed significantly lower ReHo in the right medial frontal gyrus, right superior frontal gyrus, right cluster of the precuneus and right angular gyrus, and left superior parietal lobule, and higher ReHo in the right posterior lobe of the cerebellum, right cingulate gyrus, and bilateral cluster covering the lentiform nucleus, putamen, and insula compared with GSs. Benedetti et al found that males had more right-left brain hemisphere differentiation than females..$^{40}$ Our previous sleep deprivation study found that both male and female subjects majorly represented strong unilateral between the good sleep condition and sleep deprivation condition, characterized by that altered $\mathrm{ReHo}$ areas are mainly represented in the left hemisphere, and few lower ReHo areas in the right hemisphere. ${ }^{29}$ From normal sleep status to sleep deprivation status, both male and female groups demonstrated a regular pattern of changes, ie, the unilateral tendency of ReHo regions in the brain started to disappear and these regions spread to the opposite side or bilaterally. ${ }^{29} \mathrm{In}$ our study, the altered local coherence was mainly located in the right hemisphere, suggesting that male patients with OSA had strong right-left brain hemisphere differentiation.

The most interesting finding of our study was the lower ReHo signals in the precuneus, angular gyrus, and medial frontal gyrus in patients with OSA compared with GSs. To our knowledge, these areas have been identified as being part of a default mode network that is highly active during the resting state..$^{41}$ These brain regions are thought to be involved in a group of high-level cognitive functions, variously described as visuospatial imagery, consciousness, episodic memory, executive cognitive control, and behavioral inhibition. ${ }^{42,43}$ Previous studies showed decreased resting-state functional connectivity and gray matter volume in the medial prefrontal cortex of the anterior default mode network in patients with OSA, indicating both structural and functional deficits in the medial prefrontal cortex which may be related to impaired cognitive function in these patients. ${ }^{44}$ Moreover, intermittent hypoxia is found to have a negative correlation with cerebral activation in the bilateral frontal and left parietal regions, and may be a major factor in default mode network dysfunction in patients with OSA. ${ }^{45}$ The precuneus is located in the posteromedial cortex of the parietal lobe and is a widespread network of higher association cortical and subcortical structures, and plays an important role in an array of advanced cognition functions, including visuospatial imagery, episodic memory retrieval, self-processing, and consciousness. ${ }^{42} \mathrm{~A}$ previous sleep study has shown abnormal brain function in this region. ${ }^{46}$ Positron emission tomography measurements of glucose utilization indicated that metabolism was decreased in the precuneus and prefrontal cortex. ${ }^{47}$ The functional impairment of the precuneus may have been caused partly by remote effects originating from morphologically impaired areas with decreased connectivity. ${ }^{48}$ In support of these findings, we found that patients with OSA had decreased ReHo in the precuneus 


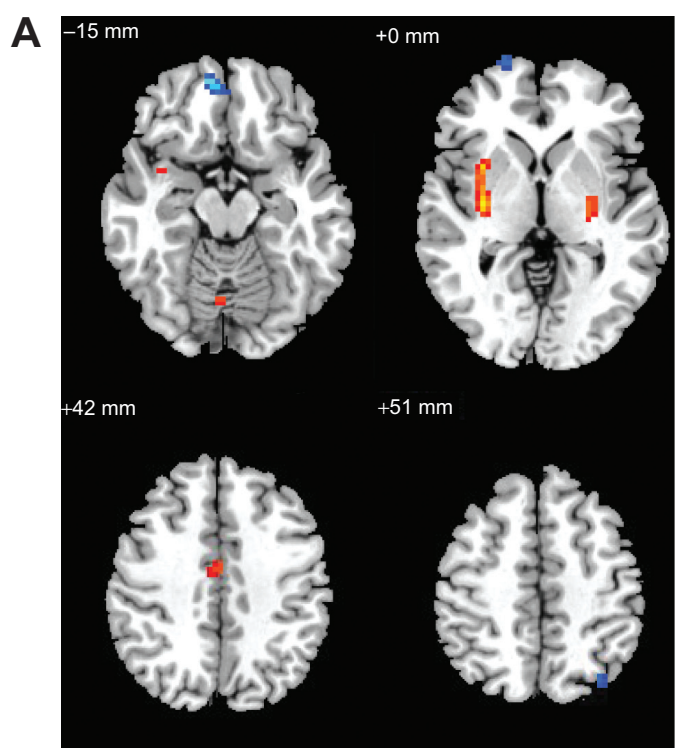

B
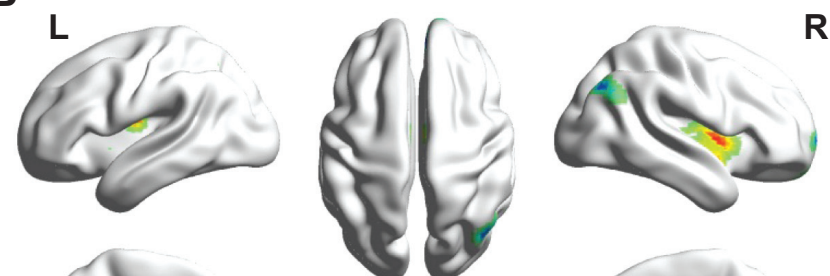

$\mathbf{R}$
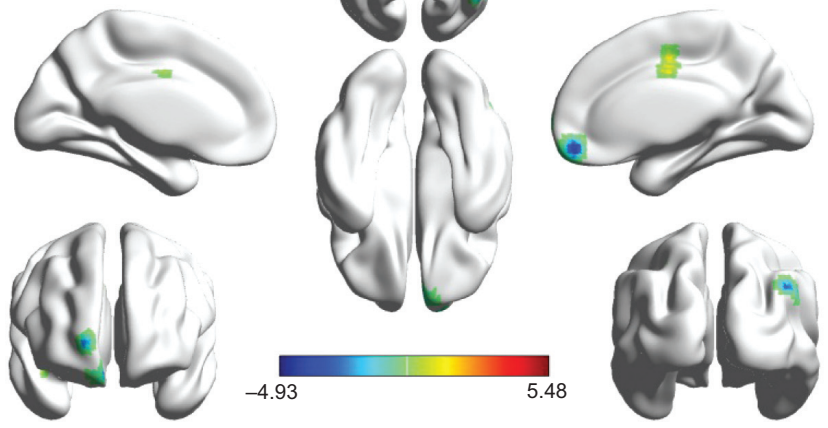

Figure I Regional homogeneity modeled in the magnetic resonance images of the subjects in the transverse orientation, shown at four different levels through the brain (A). The modeled surface of the brain shown in different orientations. The red color signifies an increase in ReHo areas, and the blue signifies a decrease in ReHo areas (B). Notes: Compared with GSs, patients with obstructive sleep apnea showed altered regional homogeneity areas, including the right medial frontal gyrus, right superior frontal gyrus, right cluster of precuneus and angular gyrus and left superior parietal lobule, right cerebellum posterior lobe, right cingulate gyrus and bilateral cluster covering the lentiform nucleus, putamen and insula.

Abbreviations: GSs, good sleepers; L, left; R, right; ReHo, regional homogeneity.

compared with GSs, and the altered ReHo in the precuneus showed a significant negative correlation with sleep time ( $r=-0.430, P=0.032$ ), suggesting decreased sleep time may

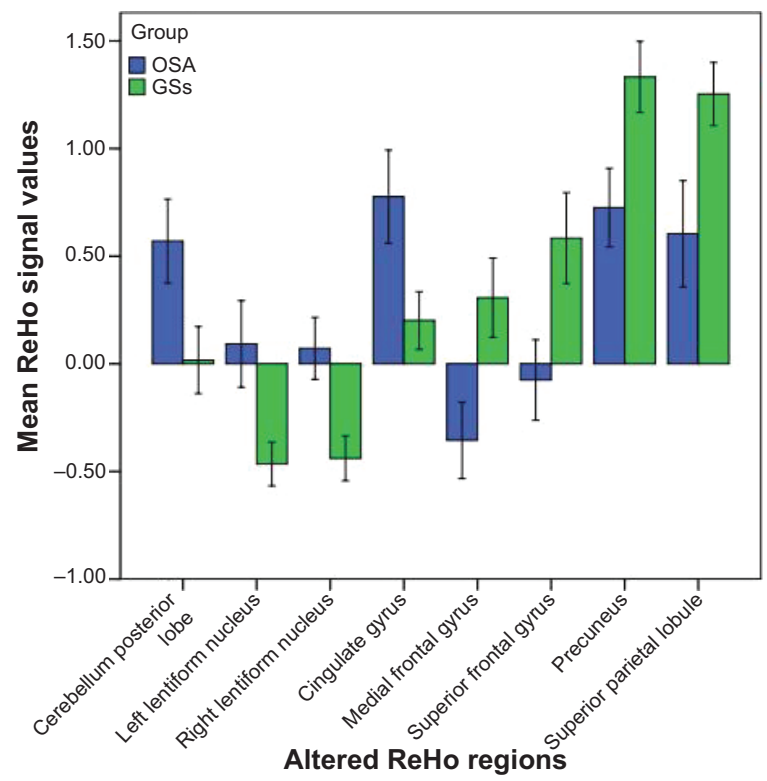

Figure 2 Mean ReHo signal values for altered regional brain areas. Patients with OSA showed altered $\mathrm{ReH}$ in the right posterior lobe of the cerebellum $(0.57 \pm 0.47$ versus $0.17 \pm 0.38)$, left lentiform nucleus $(0.93 \pm 0.49$ versus $-0.47 \pm 0.25)$, right lentiform nucleus $(0.07 \pm 0.35$ versus $-0.44 \pm 0.25)$, right cingulate gyrus $(0.78 \pm 0.52$ versus $0.20 \pm 0.32)$, right medial frontal gyrus $(-0.36 \pm 0.43$ versus $0.31 \pm 0.45)$, right superior frontal gyrus $(-0.08 \pm 0.45$ versus $0.58 \pm 0.5 \mathrm{I})$, right precuneus $(0.73 \pm 0.44$ versus I.33 \pm 0.40$)$, and left superior parietal lobule $(0.60 \pm 0.60$ versus $1.25 \pm 0.35)$ compared with GSs.

Abbreviations: GSs, good sleepers; OSA, subjects with obstructive sleep apnea; ReHo, regional homogeneity. be an important factor for dysfunction in the precuneus. Therefore, analysis of ReHo may be useful for indexing the extent of sleep time and may be an early biomarker of brain injury in patients with OSA.

Joo et al found that patients with OSA frequently showed dysregulation of respiratory control, which might be related to morphological differences in the gray matter areas of the brain, such as the superior frontal gyrus, cingulate gyrus, and left precentral gyrus. ${ }^{49}$ Previous morphological MRI studies showed significant gray matter loss or volume decrease in the frontal lobe, ${ }^{17,49}$ which may result in memory impairment, and executive dysfunctions. A previous MRS study demonstrated significant lower $\mathrm{N}$-acetylaspartate to creatine ratios in the frontal cortex and frontal white matter of patients with OSA when compared with GSs ${ }^{22}$ suggesting the presence of cerebral damage, probably caused by chronic repeated intermittent hypoxia. ${ }^{23}$ Previous task functional MRI studies in individuals with OSA showed decreased activation in the frontal cortex which was associated with both the duration of oxygen desaturation and the arousal index. ${ }^{50,51}$ In support of these findings, in our study we found that patients with OSA showed a significantly altered pattern of local cortical and subcortical activity in the right frontal gyrus (BA10, BA11) when compared with GSs.

We also found higher ReHo values in the right posterior lobe of the cerebellum, right cingulate gyrus, and bilateral cluster covering the lentiform nucleus, putamen, 
and insula in patients with OSA compared with GSs. OSA is characterized by repeated episodes of partial or complete obstruction of the upper airway during sleep. ${ }^{52}$ Evidence has shown that during respiration some cingulate neurons discharged at pace, suggesting that the anterior cingulate cortex has a complex and indirect relationship to respiratory control mechanisms. ${ }^{53}$ Moreover, it is important in the insular cortex for the perception of dyspnea. ${ }^{54}$ The insular and cingulate areas are activated by dyspnea. ${ }^{55,56}$ Cerebellar structures have been associated with regulation of respiratory patterns. ${ }^{57}$

The lentiform nucleus is involved in advanced cognitive function, and is sensitive to intermittent hypoxia with abundant blood supply, because of which patients with OSA, characterized by chronic intermittent hypoxia, showed higher ReHo in bilateral lentiform nucleus compared with GSs, which may be a compensatory to adapt to exposed to longterm hypoxia condition. Interestingly, these areas displayed symmetrical distribution, which may contribute to the about equal degree of hypoxia between two hemispheres.

\section{Conclusion}

In conclusion, our results showed altered synchrony of regional spontaneous activity in the default mode circuit during the resting-state condition. The ReHo on restingstate functional MRI is a simple and noninvasive method for investigating OSA, and is a promising tool for better understanding the neurobiological consequences of this condition. Although our results provide novel information with regard to our understanding of OSA, several limitations need to be considered. Firstly, larger sample sizes and female groups are needed in future studies to explore the mechanisms of OSA in the brain. Secondly, polysomnographic data were obtained after a single-night recording, so a first-night effect on sleep parameters could not been excluded. Thirdly, we only focused on the effect of severe OSA; other types of patients with OSA were not examined in this study.

\section{Acknowledgments}

The authors are grateful to the subjects who participated in this research. This work was supported by grants from the Jiangxi Provincial Department of Science and Technology Support Program (20132BBG70061 and 20141BBG70026), the Jiangxi Provincial Department of Natural Science Foundation Project (20132BAB205100), the Jiangxi Provincial Department of Graduate Innovation Foundation (YC2013S007), the Chinese Department of National Innovation
Experiment Program for University Students (201210403052 and 2012181).

\section{Disclosure}

The authors report no conflicts of interest in this work. None of the authors have any personal or financial involvement with organizations that have a financial interest in its content.

\section{References}

1. Park JG, Ramar K, Olson EJ, et al. Updates on definition, consequences, and management of obstructive sleep apnea. Mayo Clinic Proc. 2011; 86:549-555.

2. Punjabi NM. The epidemiology of adult obstructive sleep apnea. Proc Am Thorac Soc. 2008;5:136-143.

3. Young T, Peppard PE, Gottlieb DJ. Epidemiology of obstructive sleep apnea: a population health perspective. Am J Respir Crit Care Med. 2002; 165:1217-1239.

4. National Heart, Lung, and Blood Institute Working Group on Sleep Apnea. Sleep apnea: is your patient at risk? Am Fam Physician. 1996; 53:247-253.

5. Lumeng JC, Chervin RD. Epidemiology of pediatric obstructive sleep apnea. Proc Am Thorac Soc. 2008;5:242-252.

6. Esposito M, Antinolfi L, Gallai B, et al. Executive dysfunction in children affected by obstructive sleep apnea syndrome: an observational study. Neuropsychiatr Dis Treat. 2013;9:1087-1094.

7. Seneviratne U, Puvanendran K. Excessive daytime sleepiness in obstructive sleep apnea: prevalence, severity, and predictors. Sleep Med. 2004;5:339-343.

8. Ip MS, Lam B, Ng MM, Lam WK, Tsang KW, Lam KS. Obstructive sleep apnea is independently associated with insulin resistance. Am J Respir Crit Care Med. 2002;165:670-676.

9. Bagai K. Obstructive sleep apnea, stroke, and cardiovascular diseases. Neurologist. 2010;16:329-339.

10. Tregear S, Reston J, Schoelles K, Phillips B. Obstructive sleep apnea and risk of motor vehicle crash: systematic review and meta-analysis. J Clin Sleep Med. 2009;5:573-581.

11. Verstraeten E. Neurocognitive effects of obstructive sleep apnea syndrome. Curr Neurol Neurosci Rep. 2007;7:161-166.

12. Ennett LS, Barbour C, Langford B, Stradling JR, Davies RJ. Health status in obstructive sleep apnea: relationship with sleep fragmentation and daytime sleepiness, and effects of continuous positive airway pressure treatment. Am J Respir Crit Care Med. 1999;159:1884-1890.

13. Decary A, Rouleau I, Montplaisir J. Cognitive deficits associated with sleep apnea syndrome: a proposed neuropsychological test battery. Sleep. 2000;23:369-381.

14. Dusak A, Ursavas A, Hakyemez B, Gokalp G, Taskapilioglu O, Parlak M. Correlation between hippocampal volume and excessive daytime sleepiness in obstructive sleep apnea syndrome. Eur Rev Med Pharmacol Sci. 2013;17:1198-1204.

15. Morrell MJ, McRobbie DW, Quest RA, Cummin AR, Ghiassi R, Corfield DR. Changes in brain morphology associated with obstructive sleep apnea. Sleep Med. 2003;4:451-454.

16. Torelli F, Moscufo N, Garreffa G, et al. Cognitive profile and brain morphological changes in obstructive sleep apnea. Neuroimage. 2011; 54:787-793.

17. Canessa N, Castronovo V, Cappa SF, et al. Obstructive sleep apnea: brain structural changes and neurocognitive function before and after treatment. Am J Respir Crit Care Med. 2011;183:1419-1426.

18. Morrell M, Jackson M, Twigg G, et al. Changes in brain morphology in patients with obstructive sleep apnoea. Thorax. 2010;65:908-914.

19. Kumar R, Chavez AS, Macey PM, Woo MA, Yan-Go FL, Harper RM Altered global and regional brain mean diffusivity in patients with obstructive sleep apnea. J Neurosci Res. 2012;90:2043-2052. 
20. Macey PM, Henderson LA, Macey KE, et al. Brain morphology associated with obstructive sleep apnea. Am J Respir Crit Care Med. 2002;166: 1382-1387.

21. Macey PM, Kumar R, Woo MA, et al. Brain structural changes in obstructive sleep apnea. Sleep. 2008;31:967-977.

22. Alchanatis M, Deligiorgis N, Zias N, et al. Frontal brain lobe impairment in obstructive sleep apnoea: a proton MR spectroscopy study. Eur Respir J. 2004;24:980-986.

23. Algin O, Gokalp G, Ocakoglu G, Ursavas A, Taskapilioglu O, Hakyemez B. Neurochemical-structural changes evaluation of brain in patients with obstructive sleep apnea syndrome. Eur J Radiol. 2012; 81:491-495

24. O'Donoghue FJ, Wellard RM, Rochford PD, et al. Magnetic resonance spectroscopy and neurocognitive dysfunction in obstructive sleep apnea before and after CPAP treatment. Sleep. 2012;35:41-48.

25. Raichle ME. Two views of brain function. Trends Cogn Sci. 2010; 14:180-190.

26. Ayalon L, Ancoli-Israel S, Aka AA, McKenna BS, Drummond SPA. Relationship between obstructive sleep apnea severity and brain activation during a sustained attention task. Sleep. 2009;32:373-381.

27. Castronovo V, Canessa N, Strambi LF, et al. Brain activation changes before and after PAP treatment in obstructive sleep apnea. Sleep. 2009; 32:1161-1172.

28. Macey KE, Macey PM, Woo MA, et al. Inspiratory loading elicits aberrant fMRI signal changes in obstructive sleep apnea. Respir Physiol Neurobiol. 2006;151:44-60.

29. Dai XJ, Gong HH, Wang YX, et al. Gender differences in brain regional homogeneity of healthy subjects after normal sleep and after sleep deprivation: a resting-state fMRI study. Sleep Med. 2012;13:720-727.

30. Guo J, Chen N, Li R, et al. Regional homogeneity abnormalities in patients with transient ischaemic attack: a resting-state fMRI study. Clin Neurophysiol. 2014;125:520-525.

31. Liu H, Liu Z, Liang M, et al. Decreased regional homogeneity in schizophrenia: a resting state functional magnetic resonance imaging study. Neuroreport. 2006;17:19-22.

32. Wu T, Long X, Zang Y, et al. Regional homogeneity changes in patients with Parkinson's disease. Hum Brain Mapp. 2009;30:1502-1510.

33. Zhong Y, Lu G, Zhang Z, et al. Altered regional synchronization in epileptic patients with generalized tonic-clonic seizures. Epilepsy Res. 2011;97:83-91.

34. Santarnecchi E, Sicilia I, Richiardi J, et al. Altered cortical and subcortical local coherence in obstructive sleep apnea: a functional magnetic resonance imaging study. J Sleep Res. 2013;22:337-347.

35. Redline S, Budhiraja R, Kapur V, et al. The scoring of respiratory events in sleep: reliability and validity. J Clin Sleep Med. 2007;3:169-200.

36. [No authors listed]. EEG arousals: scoring rules and examples: a preliminary report from the Sleep Disorders Atlas Task Force of the American Sleep Disorders Association. Sleep. 1992;15:173-184.

37. Satterthwaite TD, Elliott MA, Gerraty RT, et al. An improved framework for confound regression and filtering for control of motion artifact in the preprocessing of resting-state functional connectivity data. Neuroimage. 2013;64:240-256.

38. Yan CG, Cheung B, Kelly C, et al. A comprehensive assessment of regional variation in the impact of head micromovements on functional connectomics. Neuroimage. 2013;76:183-201.
39. Zang Y, Jiang T, Lu Y, He Y, Tian L. Regional homogeneity approach to fMRI data analysis. Neuroimage. 2004;22:394-400.

40. Benedetti F, Smeraldi E. Neuroimaging and genetics of antidepressant response to sleep deprivation: implications for drug development. Curr Pharm Des. 2009;15:2637-2649.

41. Raichle ME, MacLeod AM, Snyder AZ, et al. A default mode of brain function. Proc Natl Acad Sci U S A. 2001;98:676-682.

42. Cavanna AE, Trimble MR. The precuneus: a review of its functional anatomy and behavioural correlates. Brain. 2006;129:564-583.

43. Wilson CR, Gaffan D, Browning PG, Baxter MG. Functional localization within the prefrontal cortex: missing the forest for the trees? Trends Neurosci. 2010;33:533-540.

44. Zhang Q, Wang D, Qin W, et al. Altered resting-state brain activity in obstructive sleep apnea. Sleep. 2013;36:651-659.

45. Prilipko O, Huynh N, Schwartz S, et al. Task positive and default mode networks during a parametric working memory task in obstructive sleep apnea patients and healthy controls. Sleep. 2011;34:293A-301A.

46. Dai XJ, Min YJ, Gong HH, et al. [Evaluation of the post-effect of acupuncture at Sanyinjiao (SP 6) under sleep deprivation by resting-state amplitude of low-frequency fluctuation: a fMRI study]. Zhongguo Zhen Jiu. 2012;32:47-52. Chinese.

47. Yaouhi K, Bertran F, Clochon P, et al. A combined neuropsychological and brain imaging study of obstructive sleep apnea. J Sleep Res. 2009; 18:36-48

48. Chételat G, Desgranges B, de la Sayette, et al. Dissociating atrophy and hypometabolism impact on episodic memory in mild cognitive impairment. Brain. 2003;126:1955-1967.

49. Joo EY, Tae WS, Lee MJ, et al. Reduced brain gray matter concentration in patients with obstructive sleep apnea syndrome. Sleep. 2010;33: 235-241.

50. Thomas RJ, Rosen BR, Stern CE, Weiss JW, Kwong KK. Functional imaging of working memory in obstructive sleep-disordered breathing. J Appl Physiol. 2005;98:2226-2234.

51. Zhang X, Ma L, Li S, Wang L. A functional MRI evaluation of frontal dysfunction in patients with severe obstructive sleep apnea. Sleep Med. 2011;12:335-340

52. Sanna A. Obstructive sleep apnoea, motor vehicle accidents, and work performance. Chron Respir Dis. 2013;10:29-33.

53. Frysinger R, Harper R. Cardiac and respiratory relationships with neural discharge in the anterior cingulate cortex during sleep-waking states. Exp Neurol. 1986;94:247-263.

54. Schön D, Rosenkranz M, Regelsberger J, Dahme B, Büchel C, von Leupoldt A. Reduced perception of dyspnea and pain after right insular cortex lesions. Am J Respir Crit Care Med. 2008;178:1173-1179.

55. Banzett RB, Mulnier HE, Murphy K, Rosen SD, Wise RJ, Adams L. Breathlessness in humans activates insular cortex. Neuroreport. 2000; 11:2117-2120.

56. Peiffer C, Poline JB, Thivard L, Aubier M, Samson Y. Neural substrates for the perception of acutely induced dyspnea. Am J Respir Crit Care Med. 2001;163:951-957.

57. Xu F, Frazier DT. Respiratory-related neurons of the fastigial nucleus in response to chemical and mechanical challenges. $J$ Appl Physiol. 1997;82:1177-1184.
Neuropsychiatric Disease and Treatment

\section{Publish your work in this journal}

Neuropsychiatric Disease and Treatment is an international, peerreviewed journal of clinical therapeutics and pharmacology focusing on concise rapid reporting of clinical or pre-clinical studies on a range of neuropsychiatric and neurological disorders. This journal is indexed on PubMed Central, the 'PsycINFO' database and CAS,
Dovepress

and is the official journal of The International Neuropsychiatric Association (INA). The manuscript management system is completely online and includes a very quick and fair peer-review system, which is all easy to use. Visit http://www.dovepress.com/testimonials.php to read real quotes from published authors. 